\title{
Driving Potential and Noise Level Determine the Synchronization State of Hydrodynamically Coupled Oscillators
}

\author{
Nicolas Bruot, ${ }^{1}$ Jurij Kotar, ${ }^{1}$ Filippo de Lillo, ${ }^{2,3}$ Marco Cosentino Lagomarsino, ${ }^{4,5,2}$ and Pietro Cicuta ${ }^{1}$ \\ ${ }^{1}$ Cavendish Laboratory and Nanoscience Centre, University of Cambridge, Cambridge, United Kingdom \\ ${ }^{2}$ Dipartimento di Fisica, Università di Torino, Torino, Italy \\ ${ }^{3}$ DICAT, Università di Genova, Genova, Italy \\ ${ }^{4}$ Genomic Physics Group, UMR 7238 CNRS “Microorganism Genomics," Paris, France \\ ${ }^{5}$ University Pierre et Marie Curie, Paris, France
}

(Received 11 July 2012; revised manuscript received 23 August 2012; published 19 October 2012)

\begin{abstract}
Motile cilia are highly conserved structures in the evolution of organisms, generating the transport of fluid by periodic beating, through remarkably organized behavior in space and time. It is not known how these spatiotemporal patterns emerge and what sets their properties. Individual cilia are nonequilibrium systems with many degrees of freedom. However, their description can be represented by simpler effective force laws that drive oscillations, and paralleled with nonlinear phase oscillators studied in physics. Here a synthetic model of two phase oscillators, where colloidal particles are driven by optical traps, proves the role of the average force profile in establishing the type and strength of synchronization. We find that highly curved potentials are required for synchronization in the presence of noise. The applicability of this approach to biological data is also illustrated by successfully mapping the behavior of cilia in the alga Chlamydomonas onto the coarse-grained model.
\end{abstract}

DOI: 10.1103/PhysRevLett.109.164103

PACS numbers: 05.45.Xt, 05.40.Ca, 87.80.Cc

From pacemaker cells in the heart [1,2] to laser-based communication [3], from cricket songs [4] to cells in developing embryos [5], synchronization is a central concept of science [6]. Recent work on hydrodynamics points to a new kind of synchronization [7], with experimental and theoretical evidence supporting the original hypothesis by Taylor [8] that the coordinated beating of cilia and flagella [9] is caused by the interactions through the surrounding fluid [7,10-14]. Understanding this physical problem has importance for technology as well as biology, where actively driven filaments (cilia and flagella) are ubiquitous, and key to the functionality of diverse tissues and possibly to the evolution of multicellularity. One central question is how the internal engine of cilia integrates the cues coming from the fluid in order to achieve synchronization with neighbors, or lose it when this is needed.

Current technology allows us to build micron-scale actively driven units that exhibit hydrodynamic synchronization, and that are simple to describe theoretically, allowing quantitative studies of collective dynamics [14-17]. We have developed a configuration-dependent geometricswitch feedback system to drive colloidal particles with optical traps. In this Letter, extending previous work on two [14] and more [16] driven harmonic oscillators, we show how a general form of driving potential determines the dynamical steady state in competition with thermal noise. The driving potential represents in a coarse-grained fashion the internal force engine with which the active unit pushes the fluid during each beating cycle. We relate the experimental findings to fully stochastic Brownian dynamics (BD) simulations [18], and we study the system analytically (simplifying the role of thermal noise by neglecting the coupling of thermal fluctuations).

The motion of two externally driven spherical particles at low Reynolds number is described by the force balance

$$
0=\mathbf{F}_{i}-\sum_{j} \mathbf{H}_{i, j}^{-1} \dot{\mathbf{r}}_{j}+\mathbf{f}_{i}(t),
$$

where $i \in\{1,2\}$ indexes the bead. $\mathbf{F}_{i}$ represents the driving force acting on bead $i$, and $\dot{\mathbf{r}}_{i}$ is its velocity. The drag is modeled by the Oseen tensor $\mathbf{H}$ [19] and $\mathbf{f}_{i}$ is a stochastic term describing the Brownian force on bead $i$. For the time scales considered here, the noise is adequately described by $\left\langle f_{i}(t)\right\rangle=0$ and $\left\langle f_{i}(t) f_{j}\left(t^{\prime}\right)\right\rangle=2 k_{B} T \mathbf{H}_{i, j}^{-1} \delta\left(t-t^{\prime}\right)$ [20]. We consider for simplicity only the case of driving forces $\mathbf{F}_{i}$ parallel to the direction of alignment of the particles $x$, which leads to coupling forces also directed along $x$ (aside from fluctuations). Projected along this direction, the system of equations becomes

$$
\begin{aligned}
& 0=F_{1}\left(x_{1}, t\right)-\gamma\left(\dot{x}_{1}-\epsilon \dot{x}_{2}\right)+f_{1}(t), \\
& 0=F_{2}\left(x_{2}, t\right)-\gamma\left(\dot{x}_{2}-\epsilon \dot{x}_{1}\right)+f_{2}(t),
\end{aligned}
$$

where $\epsilon=3 a /(2 d)$ is the coefficient describing coupling between the particles, $a$ is the bead radius, $d$ is the mean distance between the two beads, and $\gamma=6 \pi \eta a$ is the drag coefficient given a viscosity $\eta$. The nondiagonal terms of the Oseen tensor originate from the flow field created by one bead and acting as a drag on the other bead.

A "switch" rule maintains colloids out of equilibrium [21] [Fig. 1(a)]: the basic oscillation cycle of each bead is built by alternatively activating two traps, described by the 


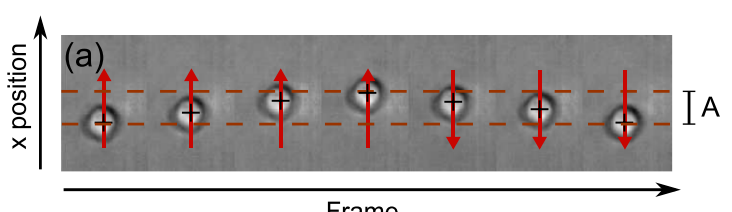

Frame

A
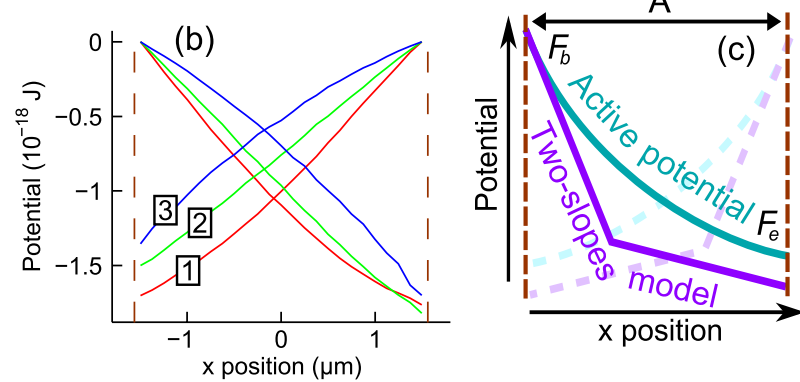

FIG. 1 (color online). (a) Selected frames showing a single bead driven by optical traps through the geometric switch rule. The direction of the gradient of laser intensity is shown by the arrows. The direction of the gradient is reversed when the particle reaches a position defined by the dashed line; the bead is maintained in oscillation. (b) Mean driving potentials for a bead driven in potentials of positive (1), null (2), and negative (3) curvature. (c) Schematic showing that a generic potential is modeled in the theory by "two slopes." The curvature parameter $c$ is measured from the mean potential in (b) by fitting the slopes at $-A / 2$ and $A / 2$, giving $F_{b}$ and $F_{e}$ for both decreasing and increasing potentials. Experimental parameters in (b): $A=$ $3.1 \mu \mathrm{m}, d=10 \mu \mathrm{m}, T=296 \mathrm{~K}, F_{0} \sim 0.53 \mathrm{pN}$, and $c=$ $0.38(1),-0.11(2)$, and $-0.40(3)$. Experiments are 2 min (single bead) and 5 min (two beads) long, while simulations in Figs. 3 and 4 are equivalent to $2000 \mathrm{~s}$.

same potential, but centered on different positions. The simulations consider potentials of the form $k_{\alpha} x^{\alpha}$, where the parameter $\alpha$ characterizes the curvature. When the first trap is active and the particle is moving towards the equilibrium position, the switching occurs when the bead reaches a position distant $s$ from the active trap center. The bead is then driven in the other direction, until it reaches the next switch, distant $s$ from the trap center (see the Supplemental Material [22]for a mathematical expression). The geometric switch imposes a constant amplitude of oscillations. Wollin and Stark [23] recently showed numerically that in a system of active oscillators, driven with the geometric-switch rule, the potential shape determines the in-phase or antiphase character of motion between nearest neighbors.

Experimentally, potential landscapes of varying curvatures are realized with time-shared optical traps, with a designed gradient of laser intensity (see the Supplemental Material [22] and Ref. [15] for details). Experimental potentials can be approximated by the power laws in simulations. More simply, their shape is captured by a parameter

$$
c=\frac{F_{b}-F_{e}}{F_{b}+F_{e}}
$$

which depends only on the forces $F_{b}$ and $F_{e}$ at the beginning and the end switch positions respectively, see Fig. 1(c). We find that all the effects due to potential shape can be related to this curvature parameter $c$ and to the mean force $F_{0}=\left(F_{b}+F_{e}\right) / 2$, as also suggested in Ref. [23] (BD simulations and the analytical calculation presented in the Supplemental Material [22] support this approximation).

Experimental potentials are created with different curvatures, as shown in Fig. 1(b). The potentials are measured from the position $x(t)$ of a single bead oscillating, by constructing a histogram of the position of the bead in time, relative to the switches of the traps. This procedure averages out the thermal noise, showing the difference of curvature in the three experiments. Experiments are conducted with $3.47 \mu \mathrm{m}$ diameter silica beads from Bangs Labs, diluted in a water-glycerol solution with a viscosity of $2.2 \mathrm{mPa}$. Trapped beads are maintained at least $70 \mu \mathrm{m}$ far from any surface of the microscope slides in order to avoid effects of wall interactions. More details about the tweezers setup are provided in Ref. [24].

These optically driven oscillators are the first experimental realization of a hydrodynamically synchronized system in which the transition between in phase and antiphase behavior is controlled simply by changing the driving forces. Numerical simulations of power law potentials are carried out, with different exponents $\alpha$ while choosing stiffnesses $k_{\alpha}$ such that the period of a cycle stays constant. These show in general synchronized dynamics, with a transition at the linear drive condition, i.e., at $\alpha=1$ $(c=0)$. Figures 2, 3(a), and 3(b) show the clear antiphase behavior observed in the steady state for potentials with positive curvature, and the in-phase behavior with negative curvature. The system with linear drive is not synchronized. A synchronization order parameter $Q(k)$ can be calculated for each half cycle $k$ of oscillation of the bead 1 [14],

$$
Q(k)=\frac{-1}{s_{k+1}-s_{k}} \int_{s_{k}}^{s_{k+1}} d t \sigma_{1}(t) \sigma_{2}(t),
$$

with $s_{k}$ the time at the $k$ th switch of bead 1 and $\sigma_{i}(t)= \pm 1$ the variable defining the state of the potential for bead $i$

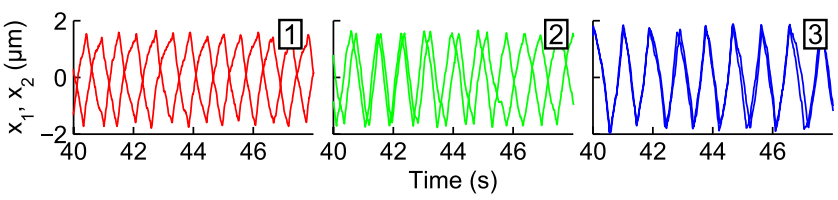

FIG. 2 (color online). Experimental tracks of two beads coupled hydrodynamically, for the three driving potentials characterized in Fig. 1(b). Positive curvature leads to antiphase (AP) synchronization while negative curvature leads to in-phase (P) synchronization. In the limit case of linear potentials (zero curvature; central section of the plot), the beads do not synchronize. 

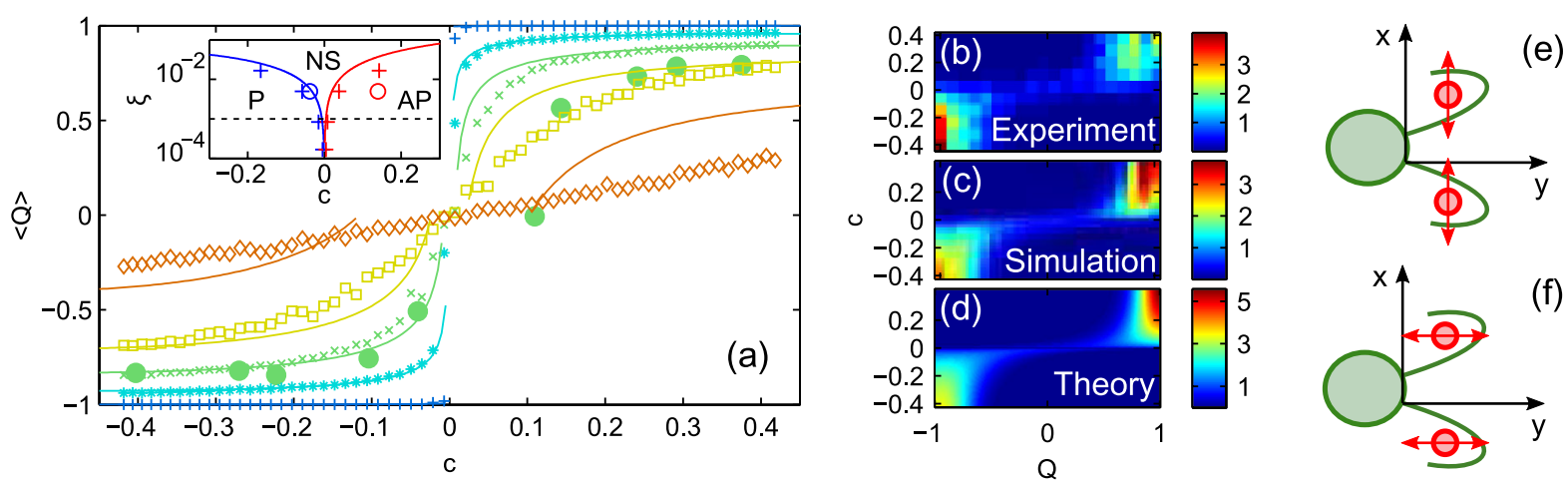

FIG. 3 (color online). (a) The phase/antiphase transition in the oscillation dynamics of the two particles is observed in experiments (○), and simulations (open markers), and is adequately captured by theory (lines). Conditions are $A=3.1 \mu \mathrm{m}$ and $F_{0} \sim 0.53 \mathrm{pN}$, and noise strength $\xi=2 k_{B} T /\left(A F_{0}\right)$ is varied by changing the temperature [from $(+)$ to $(\diamond): \xi \approx 1.68 \times 10^{-7}, 8.38 \times 10^{-4}, 4.96 \times 10^{-3}$ (room temperature), $1.68 \times 10^{-2}$, and $\left.8.38 \times 10^{-2}\right] .\langle Q\rangle$ characterizes the state of oscillations $(1$ in antiphase and -1 in in-phase oscillations). Experiments should be compared with the simulations in (). At any temperature, the state of synchronization is either in phase or in antiphase, depending on the sign the curvature $c$, with a sharper transition at lower temperature. The transition is smoother when noise is added. Theory fits very well with experiments and simulations up to room temperature. Inset: phase diagram showing synchronized states in phase (P), in antiphase (AP), or not synchronized (NS). Theory (lines), simulations $(+)$, and experiments $(\bigcirc)$ show, for each value of the noise strength $\xi$, the value of $c$ for which $|Q|=1 / 2$. The dotted line shows the noise strength estimated for Chlamydomonas. The probability distributions of $Q$ at $T=296 \mathrm{~K}$ in experiments (b), simulations (c), and from the theoretical argument (d) show that in the presence of noise, the peaks are still centered around \pm 1 but with a large spreading. The theoretical curves are interrupted close to the zero crossing, since the analytical formula fails to describe the state when synchronization is very weak or lost (see the Supplemental Material [22]). In the simulations, $s=1 \mu \mathrm{m}$. The synchronization of two beads is relevant to understanding the biflagellated organism Chlamydomonas, whose flagella perform periodic orbits in plane. The forces exerted on the fluid can be modeled as arising from a point source (a bead) and decomposed into orthogonal directions, as shown in panels (e) and (f); the consequences for synchronization are discussed in the text.

(indicates whether the driving force is pushing the bead towards increasing or decreasing positions). $Q$ is normalized so that $-1<Q<1$, with $Q=-1$ describing inphase motion and $Q=1$ anti-phase motion. As defined in Eq. (4), it is calculated for each half cycle, and its average over an experiment is $\langle Q\rangle_{k}$. As the curvature changes sign, both the experiments and the simulations show a transition from in-phase (P) to antiphase (AP) motion. In the limit of no noise (which is not accessible experimentally), we recover the known result that the pure $\mathrm{P}$ and AP motion are the only possible stable states, and the system converges to either one depending on the sign of $c$. Only for $\alpha= \pm 2$ can this result be obtained analytically, as previously shown for $\alpha=2$ [14]. However, the main question lies in the role of noise in the crossover between positive and negative curvature, and therefore requires a more general approach.

Interestingly, the general deterministic (no noise) behavior can be estimated analytically using an approximating two-slopes potential [Fig. 1(c)], which can approximate both the simulated results (power law potentials) and experimental results (where the shape is not a specific analytical function). We illustrate explicitly here the case for $c<0$, for which we expect in-phase synchronization. Consider, as a proxy for the phase, the time difference $t_{1}$ between the nearest switch of the second bead that occurs closest in time to a switch of the first bead (defined for every half cycle $k$ of one of the beads). We aim to provide an expression of $t_{1}^{(k+1)}$ depending on $t_{1}^{(k)}$. Note that $t_{1}=0$ can correspond to either an P or AP oscillation depending on the sign of $\sigma_{1}(t) \sigma_{2}(t)$ at the simultaneous switch time, but we assume here for the sake of argument an initial condition close to the in-phase state. Since in this approximation the driving potentials are only defined with constant forces, and the hydrodynamic coupling term in Eq. (2) is linear, the half cycle $k$ can be split into four parts within which the velocities of the beads will be constant at $\pm \frac{1}{\gamma}\left(F_{u} \pm \epsilon F_{v}\right)$ with $u, v \in\left\{{ }^{\prime} b^{\prime},{ }^{\prime} e^{\prime}\right\}$. Therefore, $t_{1}^{(k)}$, $t_{1}^{(k+1)}$, the start times of each part, and the positions at these times are all related by a linear system of equations that is solved in the Supplemental Material [22] and leads to a simple iterative map for $t_{1}$

$$
t_{1}^{(k+1)}=\kappa_{\mathrm{P}} t_{1}^{(k)}, \quad \text { with } \quad \kappa_{\mathrm{P}}=1+2 \epsilon \frac{F_{b}^{2}-F_{e}^{2}}{F_{e} F_{b}},
$$

to first order in the coupling coefficient $\epsilon$. For $c<0, F_{e}>$ $F_{b}$ and $\kappa_{\mathrm{P}}<1$, leading to the known result that in-phase synchronization is stable. Identical equations hold for the antiphase state with a $\kappa_{\mathrm{AP}}$ having reversed sign for $\epsilon$, and hence give stability for $c>0$. This simple model shows that the state of synchronization depends on the sign of $c$. Note that for $c=0, \kappa_{\mathrm{P}}=\kappa_{\mathrm{AP}}=1$ and the system does not synchronize. This particular case is expected, since the 
driving force over a cycle should be nonreversible in time to allow synchronization, at least in the absence of thermal fluctuations [7].

Most importantly, having approximated the driving potentials, it is possible to estimate analytically the role of noise in synchronization. Thermal noise adds fluctuations to $t_{1}$ at each half cycle, represented as a random variable $\zeta(i)$ added to Eq. (5)

$$
t_{1}^{(k+1)}=\kappa t_{1}^{(k)}+\zeta(k) .
$$

Here $\kappa$ simply represents either $\kappa_{\mathrm{P}}$ or $\kappa_{\mathrm{AP}}$ depending on the sign of $c$, allowing us to solve both $\mathrm{P}$ and AP cases at the same time. For one bead, the time difference between two consecutive switches is a first passage time. We estimate its distribution by considering each of the two linear portions of the potential separately, and neglecting the change of velocity of the beads due to the coupling term, as well as the correlations in the noise. By combining the supposed independent fluctuations for each bead (generalizing the procedure carried out in Ref. [15]), we approximate $\zeta(i)$ as a zero-mean Gaussian random variable of variance

$$
\operatorname{var}[\zeta(k)]=\frac{4 A D \gamma^{3}}{F_{0}^{3}} \frac{1+c^{2}}{\left(1-c^{2}\right)^{2}},
$$

with $D=k_{B} T / \gamma$ the diffusion coefficient of a particle. By iterating Eq. (6) we deduce the autocorrelation function of $t_{1}: g_{t_{1}}(k)=\kappa^{k} g_{t_{1}}(0)$, with $g_{t_{1}}(0)=\operatorname{var}(\zeta) /\left(1-\kappa^{2}\right)$ the variance of $t_{1} \cdot g_{t_{1}}$ is compared to simulations in Fig. 4. As $t_{1}$ is linked to the phase between the two beads, $\langle Q\rangle_{k}$ can be written in terms of $g_{t_{1}}(0)$ and the analytical function $\langle Q\rangle_{k}$ is displayed in Fig. 3(a). Further details concerning this calculation are provided in the Supplemental Material [22].

From the degree of order in the dynamics (which we take as the threshold $\left|\langle Q\rangle_{k}\right|>1 / 2$ ) it is possible to draw a phase diagram for the synchronized states as a function of the two important parameters: curvature and noise amplitude. As shown in the inset of Fig. 3(a), the analytical estimate agrees very well with the BD simulations, up to considerable levels of noise. Figure 4 shows that the simulated autocorrelation functions decay in a few cycles for highly curved potentials (large $|c|$ ), and slower near the transition, where $\kappa \rightarrow 1$.

The predictive power of the bilinear model demonstrates that the relevant feature of the internal engine affecting the synchronized state is the difference between the forces at the instances of switch. We now illustrate how this is relevant to synchronization in biological cilia and flagella, by taking as an example the biflagellated alga $C$. reinhardtii sketched in Figs. 3(e) and 3(f), where the synchronization state of the two beating filaments can be tuned, leading to different swimming modes: directed runs, and random tumbling [10]. In order to map the synchronization of the two flagella of Chlamydomonas with our coarse-grained model made of two beads, we need (a) to reconstruct the

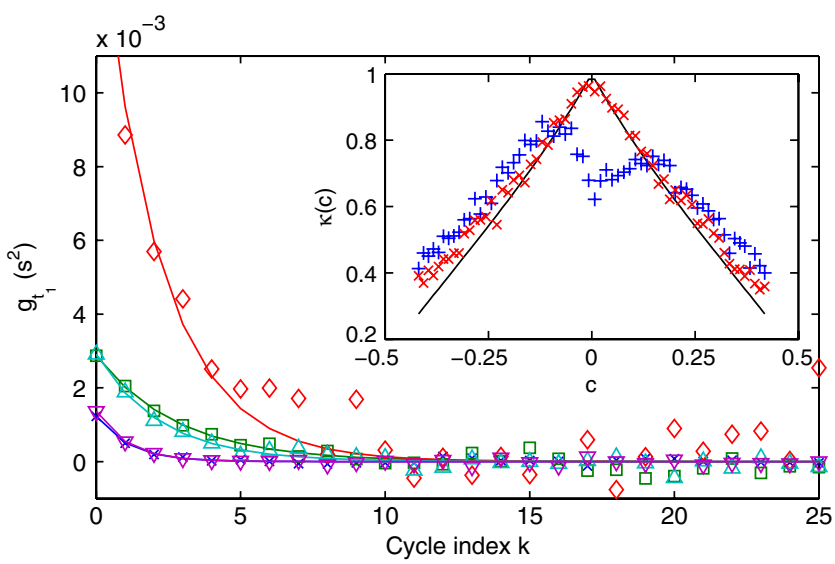

FIG. 4 (color online). Autocorrelation of the phase $t_{1}$ between the two beads in simulations for different values of $c:-0.42(\times)$, $-0.20(\square),-0.0071(\diamond), 0.20(\triangle)$, and $0.42(\nabla)$ fitted to exponential decays (solid lines). Inset: simulated decay coefficients $\kappa$ for $\xi=4.96 \times 10^{-3}(+)$ and $\xi=8.38 \times 10^{-4}(\times)$ are in good agreement with the theoretical values (line). The strength of synchronization is the strongest for the highest curvatures (high $|c|$ ). Theory and simulations differ for $c \approx 0$ because the assumption that oscillations are nearly in phase or in antiphase becomes wrong; when the noise strength increases this happens for larger values of $c$. Parameters are the same as in Fig. 3.

driving potentials of each flagellum and (b) to project the oscillations along one particular direction. This is done by using data of flagellum configurations during beating in a uniflagellated Chlamydomonas [25]. A flagellum at instant $t$ is represented by a bead located at the center of mass of the filament, subject to a driving force that is the opposite of the total force from the fluid acting on the flagellum. This model is one dimensional (i.e., the coupling forces from the Oseen tensor are in the same direction as the driving forces) in the two particular cases that we consider here: driving forces along $x$ and along $y$, with $x$ the direction of the line defined by the average position of the two beads [see Figs. 3(e) and 3(f)]. The case of oscillations along $x$ was explicitly treated up to here; the other case is described by the same equations, replacing $x$ by $y$ and changing the coupling term $\epsilon$ to $3 a /(4 d)$. Projecting the forces from Chlamydomonas into parallel and perpendicular components to the direction of motion, effective one-dimensional switching potentials can be obtained. More details on the matching of the parameters from the data on Chlamydomonas to the model are discussed in the Supplemental Material [22]. Several conclusions are found from this matching procedure: First, the mapping recovers the correct synchronized state both when projected along $x$ and $y$, i.e., flagella oscillate in phase along $y$ and in antiphase along $x$. Second, as illustrated by the dotted line in Fig. 3(a), the biological system appears quite far from the noise threshold. Third, the model predicts that the component of the hydrodynamic interaction which dominates the synchronization is $x$, i.e., 
transverse to the swimming direction of the microorganisms. This last point would be relevant in conditions where the presence of multiple organisms contributes additional periodic forces.

The phase diagram of the dynamical state versus curvature and noise enables biological systems to be mapped onto the model. This is useful in two ways: for systems (like Chlamydomonas) for which the cilia's force cycle has been measured, it is possible as outlined above to predict the state of synchronization from the coarse graining of internal degrees of freedom; on the contrary from the observation of cilia correlations at steady state it is possible to set boundaries on the cilia geometric parameters (distance, filament length) and on the average properties of the active force cycle, which result from the activity of molecular motors and the filament structure.

The geometric switch system with curved potentials has no threshold for synchronization in the absence of noise. This work shows the importance of balancing the curvature of the driving potential to the level of noise: a stronger curvature makes the steady state more robust, pointing to a requirement of time asymmetry in the driving potential for strong synchronization.

We acknowledge useful discussions with M. Polin, R.E. Goldstein, and R. Di Leonardo, and acknowledge P. V. Bayly for very kindly sharing raw data from Ref. [25]. This work was supported by the Marie Curie ITNCOMPLOIDS (FP7-PEOPLE-ITN-2008 No. 234810).

[1] C. S. Peskin, Mathematical Aspects of Heart Physiology (Courant Institute of Mathematical Sciences, New York University, New York, 1975).

[2] D. Difrancesco and P. Tortora, Nature (London) 351, 145 (1991).

[3] A. Argyris, D. Syvridis, L. Larger, V. Annovazzi-Lodi, P. Colet, I. Fischer, J. Garcia-Ojalvo, C. Mirasso, L. Pesquera, and K. Shore, Nature (London) 437, 343 (2005).

[4] J. T. Ariaratnam and S. H. Strogatz, Phys. Rev. Lett. 86, 4278 (2001).
[5] L. Herrgen, S. Ares, L. Morelli, C. Schröter, F. Jülicher, and A. Oates, Curr. Biol. 20, 1244 (2010).

[6] A. Pikovsky, M. Rosenblum, and J. Kurths, Synchonization (Cambridge University Press, Cambridge, England, 2001).

[7] R. Golestanian, J. Yeomans, and N. Uchida, Soft Matter 7, 3074 (2011).

[8] G. I. Taylor, Proc. R. Soc. A 209, 447 (1951).

[9] D. Bray, Cell Movements: From Molecules to Motility (Garland Science, New York, 2000), 2nd ed., p. 227.

[10] M. Polin, I. Tuval, K. Drescher, J. Gollub, and R. Goldstein, Science 325, 487 (2009).

[11] R. E. Goldstein, M. Polin, and I. Tuval, Phys. Rev. Lett. 103, 168103 (2009).

[12] R. E. Goldstein, M. Polin, and I. Tuval, Phys. Rev. Lett. 107, 148103 (2011).

[13] A. Vilfan and F. Jülicher, Phys. Rev. Lett. 96, 058102 (2006).

[14] J. Kotar, M. Leoni, B. Bassetti, M. Cosentino Lagomarsino, and P. Cicuta, Proc. Natl. Acad. Sci. U.S.A. 107, 7669 (2010).

[15] N. Bruot, L. Damet, J. Kotar, P. Cicuta, and M. Cosentino Lagomarsino, Phys. Rev. Lett. 107, 094101 (2011).

[16] L. Damet, G. M. Cicuta, J. Kotar, M. Cosentino Lagomarsino, and P. Cicuta, Soft Matter 8, 8672 (2012).

[17] G. M. Cicuta, E. Onofri, M. Cosentino Lagomarsino, and P. Cicuta, Phys. Rev. E 85, 016203 (2012).

[18] D. L. Ermak and J. A. McCammon, J. Chem. Phys. 69, 1352 (1978).

[19] J. Happel and H. Brenner, Low Reynolds Number Hydrodynamics: with Special Applications to Particulate Media (Kluwer, New York, 1983).

[20] M. Polin, D. G. Grier, and S. Quake, Phys. Rev. Lett. 96, 088101 (2006).

[21] M. Cosentino Lagomarsino, B. Bassetti, and P. Jona, Eur. Phys. J. B 26, 81 (2002).

[22] See Supplemental Material at http://link.aps.org/ supplemental/10.1103/PhysRevLett.109.164103 for results of simulation and analytical calculations.

[23] C. Wollin and H. Stark, Eur. Phys. J. E 34, 42 (2011).

[24] M. Leoni, J. Kotar, B. Bassetti, P. Cicuta, and M. Cosentino Lagomarsino, Soft Matter 5, 472 (2009).

[25] P. V. Bayly, B. L. Lewis, E. C. Ranz, R. J. Okamoto, R. B. Pless, and S. K. Dutcher, Biophys. J. 100, 2716 (2011). 